\title{
Synthesis and Self-Assembly of Thiol-Modified Tellurophenes
}

\begin{tabular}{|c|c|}
\hline Journal: & Canadian Journal of Chemistry \\
\hline Manuscript ID & cjc-2018-0077.R1 \\
\hline Manuscript Type: & Article \\
\hline Date Submitted by the Author: & 27-Apr-2018 \\
\hline Complete List of Authors: & $\begin{array}{l}\text { Hoover, Gabrielle ; Univeristy of Toronto, Chemistry } \\
\text { Ham, Jennifer; Univeristy of Toronto, Chemistry } \\
\text { Tang, Connie; Univeristy of Toronto, Chemistry } \\
\text { Carrera, Elisa; Univeristy of Toronto, Chemistry } \\
\text { Seferos, Dwight; Univeristy of Toronto, Chemistry }\end{array}$ \\
\hline $\begin{array}{r}\text { Is the invited manuscript for } \\
\text { consideration in a Special } \\
\text { Issue?: }\end{array}$ & Not applicable (regular submission) \\
\hline Keyword: & $\begin{array}{l}\text { tellurophene, thiol-modification, organometallic ligand, monolayer, } \\
\text { electrochemistry }\end{array}$ \\
\hline
\end{tabular}




\section{Synthesis and Self-Assembly of Thiol-Modified Tellurophenes}

Gabrielle C. Hoover, Jennifer Ham, Connie Tang, Elisa I. Carrera, and Dwight S. Seferos

Department of Chemistry, University of Toronto, Ontario, Canada, M5S 3H6

Corresponding Author: Dwight S. Seferos, E-mail: dseferos@chem.utoronto.ca; Tel: 416-9460285 


\begin{abstract}
An asymmetric thiol-modified tellurophene was designed and synthesized, and the ability of the compound to form a monolayer on a gold electrode was confirmed. The surface-active tellurophene was synthesized using Cadiot-Chodkiewicz coupling followed by ring closing and thiol modification. The tellurophene compound forms a monolayer on a gold surfaces from a concentrated solution within 24 hours. The ability of the compound to conjugate to gold is confirmed by X-ray photoelectron spectroscopy (XPS). A surface blocking experiment is then used to evaluate the extent of formation of a monolayer on a gold electrode.

Keywords: tellurophene, thiol-modification, organometallic ligand, monolayer, electrochemistry, x-ray photoelectron spectroscopy
\end{abstract}




\section{Introduction}

Modification of metal surfaces with organic molecules has received widespread attention in the physical sciences for decades because the covalent addition of ligands can impart unique electronic and physical properties, colloidal stability, and functionality. ${ }^{1-4}$ Expanding the range of possible chemical modifications to surfaces is an important area in many disciplines including nanomaterials $^{5-7}$ and medicine. ${ }^{8-11}$ Self-assembled monolayers (SAMs) of organic ligands are well-organized, well-packed, and immobilized when conjugated directly to a metal surface, and electroactive ligands will relay signals to the metal upon structural changes. ${ }^{12}$ The modification of planar metal surfaces for applications in electrochemical analysis of local environments has led to the development of small molecule ${ }^{13,14}$ and biological ${ }^{15,16}$ sensors that can provide information about analyte presence and concentration through a conductive metal. The exploration of tethering ligands with different functional groups and chemical properties is highly important to expand the library of detectable analytes, make devices more sensitive and accurate, and reduce their cost.

Tellurium, the fifth-row member of the chalcogen family, has unique chemical properties that would be valuable as part of a metal-ligand system. Its capabilities as a sensor when incorporated into various inorganic nanocrystalline assemblies such as cadmium, ${ }^{17-21}$ copper, ${ }^{22,23}$ nickel, and cobalt ${ }^{24}$ have been studied in the past several years. Despite this, tellurium has yet to be examined in organometallic or organic ligand-metal systems. Its expanded valence, metalloid nature, and ability to exist in multiple oxidation states as a part of an organometallic molecule have found applications in biotechnology and a wide range of electronic devices. ${ }^{25,26}$ When incorporated into a 5-membered tellurophene ring, tellurium can undergo reversible oxidation with quantum yields up to $42 \%,{ }^{27-29}$ which could assist in hydrogen production from hydrogen 
halides. ${ }^{30}$ Although tellurophenes have been investigated for their potential in optoelectronic materials including field effect transistors, ${ }^{31}$ photovoltaics ${ }^{32,33}$ electrochromic materials, ${ }^{34}$ and luminogens, ${ }^{35,36}$ they are still relatively unexplored compared to their sulfur and selenium analogues. Therefore, the ongoing exploration of these unique metalloid-containing heterocycles, including their synthesis and polymerization, is important. ${ }^{37-47}$ Additionally, thiophene and polythiophene derivatives have been directly conjugated to metal surfaces through thiosulfate and thiol linkers, ${ }^{48}$ demonstrating the interest in chalcogenophenes for these materials. Given the unique chemistry and potential applications of both small molecule and polymeric tellurophenes, and considering that tellurophene derivatives have been shown to function as sensors of oxygen and multidentate anionic species, ${ }^{49,50}$ developing a route to a solid-state detection system expands the environments in which a tellurophene-based sensor can be used. The ability to tether a tellurophene to a metal will also contribute to the library of metal-ligand systems and nanostructured assemblies.

In order to tether a tellurophene to gold, a commonly examined metal surface, a pendant thiol is required. Although a wide variety of substituted tellurophenes including thioesters ${ }^{51}$ have been synthesized, the lack of synthetic methods to access thiol-modified tellurophenes has prevented tellurophenes from being tethered to gold surfaces thus far. To our knowledge, there has been only one report of a thiol-containing tellurophene, in which a cysteine group recognizes $\beta$-galactosidase as part of a tellurophene mass cytometry probe. ${ }^{52}$ Multiple routes to symmetrical tellurophenes are well developed ${ }^{53,54}$ and may be applied to synthesize a thiol-modified tellurophene. Symmetry is undesirable in this study as the molecule must bond to the gold on only one end while the other end extends to face the solution; therefore, an alternative synthetic route is required. 
Here we report the synthesis of a thiol-modified tellurophene and its self-assembly into a monolayer on a gold electrode. This work expands the scope for the synthesis of asymmetric tellurophenes, specifically and importantly the installation of a thiol group which is required for their investigation as SAMs.

\section{Experimental}

\subsection{General Considerations}

All chemical reagents were obtained from Sigma Aldrich and used as received unless otherwise stated. All dry solvents were obtained using an Inert Technology solvent purification system. ${ }^{1} \mathrm{H}$ and ${ }^{13} \mathrm{C}$ NMR spectra were obtained using a Varian Mercury $400(400 \mathrm{mHz})$ spectrometer in $\mathrm{CDCl}_{3}$ with TMS as an internal standard, ${ }^{125} \mathrm{Te}$ NMR spectra were obtained using an Agilent DD2-600 (600 mHz) spectrometer in $\mathrm{CDCl}_{3}$ with TMS as an internal standard, and mass spectrometry was performed using a Waters GCT Premier TOF mass spectrometer in electron ionization mode. Optical properties of the thiol modified tellurophene were measured using a Varian Cary 5000 UV-vis-NIR spectrophotometer in double-beam mode in a quartz cuvette. Electrodes and their polishing supplies were purchased from $\mathrm{CH}$ Instruments.

\subsection{Electrochemistry}

Electrochemical measurements were performed using a BASi Epsilon potentiostat. All cyclic voltammetry experiments were performed using a three-electrode cell under ambient conditions, and all potentials were referenced to an internal $\mathrm{Ag} / \mathrm{Ag}^{+}$standard. Gold button electrodes were used to determine the ability of the ligand to modify the metal surface in MilliQ purified water. All experiments used a platinum mesh counter electrode with a surface area greater than that of the working electrode. Measurements performed in MilliQ purified water used an $\mathrm{Ag} / \mathrm{AgCl}$ reference electrode in a porous compartment filled with freshly prepared $1 \mathrm{M}$ 
$\mathrm{KCl}$ that saturated overnight before use. Fresh electrolyte solutions were prepared from $0.1 \mathrm{M}$ $\mathrm{K}_{3} \mathrm{Fe}(\mathrm{CN})_{6}$ with $1 \mathrm{M} \mathrm{KCl}$ as a supporting salt in MilliQ purified water.

Before use, gold electrode surfaces were cleaned by polishing with $0.05 \mu \mathrm{m}$ alumina powder, rinsing with MilliQ purified water, and drying with a stream of argon. To form a selfassembled monolayer, the gold electrode was submerged overnight in a covered solution of the tellurophene ligand dissolved in chloroform. The electrode was then rinsed repeatedly with chloroform to wash away any ligand that was not covalently adsorbed, rinsed with MilliQ purified water, and dried with a stream of argon.

\subsection{X-Ray Photoelectron Spectroscopy}

Silicon wafers with $50 \mathrm{~nm}$ of gold were cut into strips using a diamond scribe. The substrates were submerged overnight in a covered concentrated solution of the tellurophene ligand in chloroform. Spectra were collected using a ThermoFisher Scientific K-Alpha XPS.

\section{Results and Discussion}

\subsection{Molecular Design}

The asymmetrical tellurophene molecule was designed to have a terminal thiol and a hydrophilic solubilizing group on opposite ends. A bi-dentate ligand would form an arc around the gold surface, blocking additional ligands from adsorbing underneath it while still allowing small molecule analytes to reach the gold surface. A single thiol on one terminus is therefore crucial for the formation of a full monolayer. It is important to note that it is not necessary for the free ligand to be water-soluble, but for the ligand to extend outward in aqueous solution while tethered to the gold electrode. This will prevent the ligand from collapsing along the metal or coiling into itself, better allowing the tellurophene to interact with its local environment. Additionally, if a monolayer has not fully formed, an extended ligand will allow small analytes 
to approach the metal while a ligand collapsed on the surface will block them. Therefore, this solubilizing segment ensures equal surface blockage in all solvents.

Although tellurophene offers advantages over thiophene and selenophene, such as its reversible oxidation, it is less aromatic with shorter carbon-chalcogen bonds, less stable, ${ }^{55}$ and more susceptible to degradation. Substituting the 2 and 5 positions with phenyl groups extends the conjugation length ${ }^{56}$ and alleviates this issue. A minimum of one phenyl spacer group is therefore desirable on the side of the solubilizing group to promote ligand stability. Synthetic routes to a ligand that includes a second phenyl group on the thiol side of the tellurophene were considered to further increase its stability. Routes without a second phenyl group were also considered because increased distance between the electroactive site and the electrode decreases the efficiency of direct electron transfer. ${ }^{57}$ The synthesized ligand meeting each of the above requirements and its conjugation to a gold electrode is depicted in Figure 1.

\subsection{Synthesis}

The first consideration was to choose a synthetic route that can access an asymmetric tellurophene. Stille coupling and ipso-arylation were considered as they can result in asymmetric tellurophenes if molar ratios are carefully controlled. ${ }^{58,59}$ Alkyne coupling followed by a ring closing reaction with tellurium was preferable due to lower toxicity, higher stability, and more facile purification. An asymmetric Cadiot-Chodkiewicz coupling using a copper (I) catalyst was selected to first give an asymmetric diyne that could undergo ring-closing. This required the synthesis of two alkyne coupling partners, one of which is brominated. Using methyl triethylene glycol as the solubilizing agent, modified alkyne $\mathbf{2}$ was accessible in two steps. It was then chosen as the brominated coupling partner due to the ease of its synthesis. The synthesis of the 
brominated solubilizing and stabilizing alkyne $\mathbf{3}$ was therefore accomplished in three steps from 4-iodophenol (Scheme 1).

To reduce the number of linear synthetic steps, we first attempted to synthesize a second alkyne coupling partner containing a thioacetate and planned to reduce it to a thiol during the self-assembly process. This would allow tellurium insertion to be the last synthetic step. Attempts to synthesize a thioacetate with a terminal alkyne began with a substitution of the proton of 1 with 1,3-dibromo propane to form 8 (Scheme 2). Following alkyne deprotection to form 9 , the resulting terminal bromine could be substituted with thioacetate to form $\mathbf{1 0}$. Attempts to deprotect the alkyne in THF and methanol failed, so $\mathbf{9}$ and $\mathbf{1 0}$ were inaccessible by these methods. A second phenyl group was predicted to be disadvantageous for electron transfer; therefore, all other synthetic routes used a short, flexible alkyl chain between the tellurophene and thiol to minimize the distance between the tellurophene heterocycle and the gold surface. A successful route to a thioacetate containing alkyne $\mathbf{1 1}$ was found by substituting propargyl bromide with potassium thioacetate in 75\% yield. Cadiot-Chodkiewicz coupling between 11 and 3 was unsuccessful, likely due to sulfur poisoning the copper catalyst. ${ }^{60}$ This led us to conclude that the alkyne coupling must occur before the thiol modification.

In our adopted synthetic pathway that allowed us to access a thiol-modified asymmetric tellurophene, 1-butynol was coupled with 3 to produce a diyne with a terminal alcohol (4). Here, two routes to access the final tellurophene were considered. The first was through a tellurium ring closing reaction followed by thioacetate substitution using a Mitsonobu reaction. The second was through the Mitsonubu reaction first, then forming the tellurophene ring with a thioacetate group present. It was more likely that the tellurophene would be tolerant to Mitsonobu conditions than a thioacetate or thiol would be to tellurium ring closing conditions; 
therefore, the ring closing reaction was performed next (Scheme 3). Compound 5 was formed in a $60 \%$ yield which was then taken forward to form the thioacetate $\mathbf{6}$. The use of DIAD resulted in the formation of a high-mass side product that had the same solubility and co-eluted with $\mathbf{6}$ using all attempted purification methods, preventing isolation of the thioacetate for self-assembly studies (Figure S1). Reducing the thioacetate to thiol 7 caused it to lose its solubility in methanol, allowing it to be isolated from the methanol-soluble impurity by repeated washing with solvent. Although some compounds were formed in poor yield (Table 1), a route to thiol-modified tellurophenes was discovered.

\subsection{Optical Characterization}

Most tellurophene derivatives that are substituted with phenyl groups in the 2 and 5 positions have maximum optical absorbance between 330 and $365 \mathrm{~nm}$ with extinction coefficients between 21,300 and $31,200 \mathrm{~L} \mathrm{~mol}^{-1} \mathrm{~cm}^{-1} \cdot{ }^{29}$ In contrast, tellurophene 7 has a maximum absorbance at $309 \mathrm{~nm}$ and an extinction coefficient of 14,000 $\mathrm{L} \mathrm{mol}^{-1} \mathrm{~cm}^{-1}$. Both of these differences from previously reported compounds were attributed to the tellurophene being substituted with one phenyl and one alkyl chain rather than two phenyl groups. This decreased the conjugation length and caused the observed blue-shift as well as a decrease in extinction coefficient.

\subsection{Monolayer Formation}

To confirm the tellurophene ligand is able to conjugate to a gold electrode, XPS may be used to confirm the presence of tellurium on a gold-coated silicon substrate. A control substrate was submerged in chloroform and covered overnight to provide a baseline for comparison. The experimental substrate was submerged in a concentrated solution of the tellurophene ligand in chloroform and covered overnight. After monolayer formation, the Te elemental composition by 
atomic $\%$ increased from $0 \%$ to $2.18 \%$ at the Te $3 \mathrm{~d}$ peak at $573.65 \mathrm{eV}$ (Figure 3 ). Additionally, the $\mathrm{Au} 4 \mathrm{f}$ peak at $83.99 \mathrm{eV}$ decreased from $44.27 \%$ to $1.88 \%$, indicating the increased presence of other elements on the surface (Figure S12). The presence of tellurium and the decrease in the elemental gold atomic \% confirm the ability of the ligand to conjugate to gold surfaces.

Next, an electrochemical assay was used to determine that a robust monolayer had been formed. ${ }^{61}$ As a control, a bare gold electrode was submerged in $0.1 \mathrm{M} \mathrm{K}_{3} \mathrm{Fe}(\mathrm{CN})_{6}$ with $1 \mathrm{M} \mathrm{KCl}$ in water, and the $\left[\mathrm{Fe}(\mathrm{CN})_{6}\right]^{-3 /-4}$ redox couple was examined to provide a baseline cyclic voltammetry measurement. As the potential was swept positively to $+0.6 \mathrm{~V}$ and negatively to $0.2 \mathrm{~V}$, we observed peak oxidation and reduction currents of 0.5 and $-0.6 \mathrm{~mA}$ respectively with a half wave potential of $+0.262 \mathrm{~V}$ over three cycles under ambient conditions. (Figure 2).

Following the submersion of a freshly polished and clean gold electrode in a tellurophene ligand solution overnight, cyclic voltammetry was used to examine the extent of self-assembled monolayer formation. It was expected that the monolayer will fully cover the surface of the gold from the outside, preventing the gold electrode from interacting with $\mathrm{K}_{3} \mathrm{Fe}(\mathrm{CN})_{6}$. Therefore, it was expected that significantly less electrochemical activity will be observable by a cyclic voltammetry measurement. This was indeed our observation; oxidation and reduction peaks were reduced after the electrode was soaked for 24 hours in a dilute ligand solution and were indistinguishable after soaking for 24 hours in a concentrated solution, forming a complete monolayer. At the expected half-wave potential of $+0.262 \mathrm{~V}$, the currents were below $100 \mu \mathrm{A}$ after 24 hours of submersion of the gold electrode in a concentrated solution of the tellurophene ligand. The small observable current values were likely due to residual non-Faradaic current rather than incomplete surface coverage; otherwise, distinct oxidation and reduction peaks would still be observable. The disappearance of oxidation and reduction peaks as well as the decrease in 
current confirms a monolayer of tellurophene ligand formed on the gold surface of the electrolyte.

\section{Conclusion}

An asymmetrical thiol-modified tellurophene was synthesized through CadiotChodkiewicz coupling of alkynes. Multiple synthetic routes were explored, and alkyne coupling followed by tellurium insertion, thioacetate substitution, and reduction to a thiol was a successful approach to synthesizing a thiol-modified tellurophene. The reported ligand has a higher energy absorption and lower extinction coefficient than tellurophenes disubstituted with phenyl derivatives due to the lower conjugation length. The ability of the tellurophene ligand to conjugate to gold was confirmed using XPS, and formation of a monolayer on the surface of a gold electrode was confirmed with a cyclic voltammetry blocking experiment. This work allows for the expansion of the library of self-assembled materials and development of tellurophene probes and sensors using metal-ligand systems.

\section{Acknowledgements}

This work was supported by the NSERC of Canada (Discovery Grant and Strategic Project Grant with 1-Material and Digital Specialty Chemicals), the CFI, and the Ontario Research Fund. The authors thank Dr. Rana Sodhi and the Ontario Centre for the Characterization of Advanced Materials in the Department of Chemical Engineering and Applied Chemistry and Materials Science Engineering for XPS analysis. G. C. Hoover is grateful for the Connaught Scholarship for International Students.

\section{References}

(1) Zhou, J.; Ralston, J.; Sedev, R.; Beattie, D. A. J Colloid Interface Sci 2009, 331 (2), 251.

(2) Vericat, C.; Vela, M. E.; Corthey, G.; Pensa, E.; Cortés, E.; Fonticelli, M. H.; Ibañez, F.; 
Benitez, G. E.; Carro, P.; Salvarezza, R. C. RSC Advances 2014, 4 (53), 27730.

(3) Janata, J.; Josowicz, M. Nat Mater 2002, 2, 19.

(4) Shreiber, F. Progress in Surface Science 2000, 65, 151.

(5) Zotti, G.; Vercelli, B.; Berlin, A. Acc. Chem. Res. 2008, 41 (9), 1098.

(6) Casalini, S.; Bortolotti, C. A.; Leonardi, F.; Biscarini, F. Chemical Society Reviews 2016, 46, 40 .

(7) Biju, V. Chem. Soc. Rev. 2014, 43 (3), 744.

(8) Bowman, M.-C.; Ballard, T. E.; Ackerson, C. J.; Feldheim, D. L.; Margolis, D. M.; Melander, C. J. Am. Chem. Soc. 2008, 130 (22), 6896.

(9) Giljohann, D. A.; Seferos, D. S.; Daniel, W. L.; Massich, M. D.; Patel, P. C.; Mirkin, C. A. Angew. Chem. Int. Ed. Engl. 2010, 49 (19), 3280.

(10) Zhang, X.-Q.; Xu, X.; Bertrand, N.; Pridgen, E.; Swami, A.; Farokhzad, O. C. Advanced Drug Delivery Reviews 2012, 64 (13), 1363.

(11) Geszke-Moritz, M.; Moritz, M. Materials Science \& Engineering C 2013, 33 (3), 1008.

(12) Zhang, S.; Cardona, C. M.; Echegoyen, L. Chem. Commun. 2006, 15 (43), 4461.

(13) Xiao, Y.; Ju, H.-X.; Chen, H.-Y. Analytica Chimica Acta 1999, 391 (1), 73.

(14) Wang, L.; Bai, J.; Huang, P.; Wang, H.; Zhang, L.; Zhao, Y. Electrochemistry Communications 2006, 8 (6), 1035.

(15) Love, J. C.; Estroff, L. A.; Kriebel, J. K.; Nuzzo, R. G.; Whitesides, G. M. Chem. Rev. 2005, 105 (4), 1103.

(16) Gooding, J. J. Electroanalysis 2002, 14 (17), 1149.

(17) Qin, Q.; Bai, X.; Hua, Z. J. Electrochem. Soc. 2017, 164 (4), H241.

(18) Kjällman, T. H. M.; Peng, H.; Soeller, C.; Travas-Sejdic, J. Analyst 2010, 135 (3), 488. 
(19) Hao, N.; Jiang, L.; Qian, J.; Wang, K. JEAC 2016, 781 (C), 332.

(20) Yang, R.; Miao, D.; Liang, Y.; Qu, L.; Li, J.; de B Harrington, P. Electrochimica Acta 2015, 173,839 .

(21) Yang, M.; Wang, Y.; Wang, H. Electrochimica Acta 2015, 169, 7.

(22) Bobrowski, A.; Królicka, A.; Śliwa, J.; Zarębski, J. Electrochimica Acta 2017, 252, 453.

(23) Xiao, G.; Zeng, Y.; Jiang, Y.; Ning, J.; Zheng, W.; Liu, B.; Chen, X.; Zou, G.; Zou, B. Small 2012, $9(5), 793$.

(24) Pradhan, S.; Das, R.; Biswas, S.; Das, D. K.; Bhar, R.; Bandyopadhyay, R.; Pramanik, P. Electrochimica Acta 2017, 238, 185.

(25) Chivers, T.; Laitinen, R. S. Chemical Society Reviews 2015, 44, 1725.

(26) Poon, J.-F.; Yan, J.; Singh, V. P.; Gates, P. J.; Engman, L. J. Org. Chem. 2016, 81 (24), 12540.

(27) Carrera, E. I.; McCormick, T. M.; Kapp, M. J.; Lough, A. J.; Seferos, D. S. Inorg Chem 2013, $52(23), 13779$.

(28) Carrera, E. I.; Seferos, D. S. Dalton Trans 2015, 44 (5), 2092.

(29) Carrera, E. I.; Lanterna, A. E.; Lough, A. J.; Scaiano, J. C.; Seferos, D. S. J. Am. Chem. Soc. 2016, $138(8), 2678$.

(30) Lin, T.-P.; Gabbaï, F. P. J. Am. Chem. Soc. 2012, 134 (29), 12230.

(31) Jahnke, A. A.; Yu, L.; Coombs, N.; Scaccabarozzi, A. D.; Tilley, A. J.; DiCarmine, P. M.; Amassian, A.; Stingelin, N.; Seferos, D. S. Journal of Materials Chemistry C 2015, $3(15), 3767$.

(32) Lv, L.; Wang, X.; Wang, X.; Yang, L.; Dong, T.; Yang, Z.; Huang, H. ACS Appl. Mater. Interfaces 2016, 8 (50), 34620. 
(33) Yang, L.; Gu, W.; Lv, L.; Chen, Y.; Yang, Y.; Ye, P.; Wu, J.; Hong, L.; Peng, A.; Huang, H. Angew. Chem. Int. Ed. 2018, 57 (4), 1096.

(34) Pander, P.; Motyka, R.; Zassowski, P.; Lapkowski, M.; Swist, A.; Data, P. J. Phys. Chem. C 2017, 121 (21), 11027.

(35) Torres Delgado, W.; Braun, C. A.; Boone, M. P.; Shynkaruk, O.; Qi, Y.; McDonald, R.; Ferguson, M. J.; Data, P.; Almeida, S. K. C.; Aguiar, I. de; de Souza, G. L. C.; Brown, A.; He, G.; Rivard, E. ACS Appl. Mater. Interfaces 2017, acsami.7b11628.

(36) Braun, C. A.; Zomerman, D.; de Aguiar, I.; Qi, Y.; Delgado, W. T.; Ferguson, M. J.; McDonald, R.; de Souza, G. L. C.; He, G.; Brown, A.; Rivard, E. Faraday Discussions 2017, 196, 255.

(37) Kumar, S.; Lee, W.-Z.; Ravikanth, M. Org. Lett. 2018, acs.orglett.7b03715.

(38) Chen, S.-Y.; Pao, Y.-C.; Sahoo, S. K.; Huang, W.-C.; Lai, Y.-Y.; Cheng, Y.-J. Chem. Commun. (Camb.) 2018, 1.

(39) Al-Rubaie, A. Z.; Yosif, M. Y.; Asker, K. A. Heteroatom Chem 2017, 28 (6), e21402.

(40) Okuma, K.; Yahata, S.; Nagahora, N.; Shioji, K. Chem. Lett. 2017, 46 (3), 405.

(41) Park, Y. S.; Wu, Q.; Nam, C.-Y.; Grubbs, R. B. Angew. Chem. Int. Ed. 2014, 53 (40), 10691.

(42) Jahnke, A. A.; Djukic, B.; McCormick, T. M.; Buchaca Domingo, E.; Hellmann, C.; Lee, Y.; Seferos, D. S. J. Am. Chem. Soc. 2013, 135 (3), 951.

(43) Jahnke, A. A.; Howe, G. W.; Seferos, D. S. Angew. Chem. Int. Ed. Engl. 2010, 49 (52), 10140.

(44) He, G.; Kang, L.; Torres Delgado, W.; Shynkaruk, O.; Ferguson, M. J.; McDonald, R.; Rivard, E. J. Am. Chem. Soc. 2013, 135 (14), 5360. 
(45) González, D. M.; Raftopoulos, K. N.; He, G.; Papadakis, C. M.; Brown, A.; Rivard, E.; Müller-Buschbaum, P. Macromol. Rapid Commun. 2017, 38 (11), 1700065.

(46) Zhang, K.; Lv, L.; Wang, X.; Mi, Y.; Chai, R.; Liu, X.; Shen, G.; Peng, A.; Huang, H. ACS Appl. Mater. Interfaces 2018, 10 (2), 1917.

(47) Ye, S.; Steube, M.; Carrera, E. I.; Seferos, D. S. Macromolecules 2016, 49 (5), 1704.

(48) Mishra, A.; Ma, C.-Q.; Bauerle, P. Chem. Rev. 2009, 109 (3), 1141.

(49) Kaur, M.; Da Seul Yang; Choi, K.; Cho, M. J.; Choi, D. H. Dyes and Pigments 2014, $100(\mathrm{C}), 118$.

(50) Garrett, G. E.; Carrera, E. I.; Seferos, D. S.; Taylor, M. S. Chem. Commun. (Camb.) 2016, 52, 9881 .

(51) Zeni, G. Tetrahedron Letters 2005, 46 (15), 2647.

(52) Lumba, M. A.; Willis, L. M.; Santra, S.; Rana, R.; Schito, L.; Rey, S.; Wouters, B. G.; Nitz, M. Org. Biomol. Chem. 2017, 15 (30), 6388.

(53) Torres Delgado, W.; Shahin, F.; Ferguson, M. J.; McDonald, R.; He, G.; Rivard, E. Organometallics 2016, 35 (12), 2140.

(54) Sathyamoorthy, B.; Axelrod, A.; Farwell, V.; Bennett, S. M.; Calitree, B. D.; Benedict, J. B.; Sukumaran, D. K.; Detty, M. R. Organometallics 2010, 29 (15), 3431.

(55) Mahrok, A. K.; Carrera, E. I.; Tilley, A. J.; Ye, S.; Seferos, D. S. Chem. Commun. (Camb.) 2015, $51(25), 5475$.

(56) McCormick, T. M.; Jahnke, A. A.; Lough, A. J.; Seferos, D. S. J. Am. Chem. Soc. 2012, $134(7), 3542$.

(57) Liu, B.; Bard, A. J.; Mirkin, M. V.; Creager, S. E. J. Am. Chem. Soc. 2004, 126 (5), 1485. 
(58) Sweat, D.; Stephens, C. Synthesis 2009, 2009 (19), 3214.

(59) Terao, Y.; Wakui, H.; Nomoto, M.; Satoh, T.; Miura, M.; Nomura, M. J. Org. Chem. 2003, $68(13), 5236$.

(60) Argyle, M.; Bartholomew, C. Catalysts 2015, 5 (1), 145.

(61) Nakaminami, T.; Ito, S.-I.; Kuwabata, S.; Yoneyama, H. Anal. Chem. 1999, 71 (19), 4278. 


\section{Tables}

Table 1. Yields of compounds in the synthesis of a thiol-modified tellurophene.

\begin{tabular}{ll}
\hline Compound & Isolated Yield (\%) \\
\hline 1 & 85 \\
2 & 41 \\
3 & 96 \\
4 & 23 \\
5 & 60 \\
6 & Not determined \\
7 & 13 \\
8 & 29 \\
11 & 75 \\
\hline
\end{tabular}


(a)

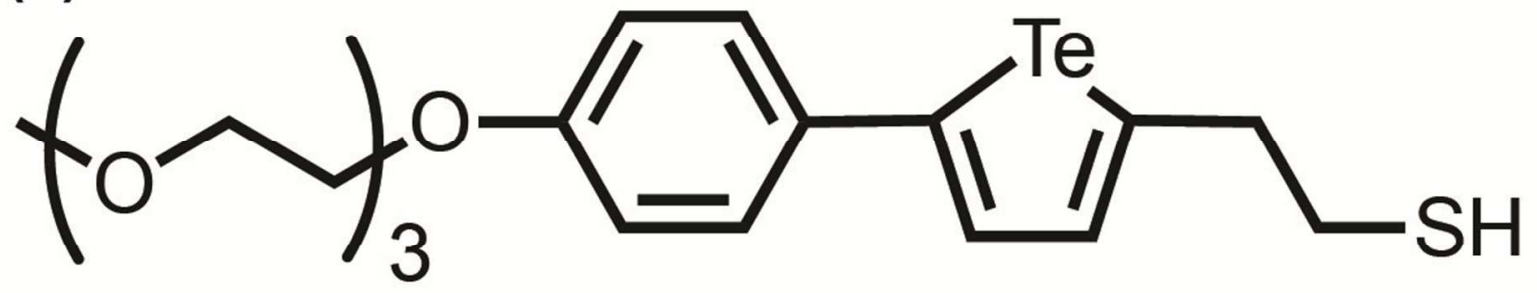

(b)

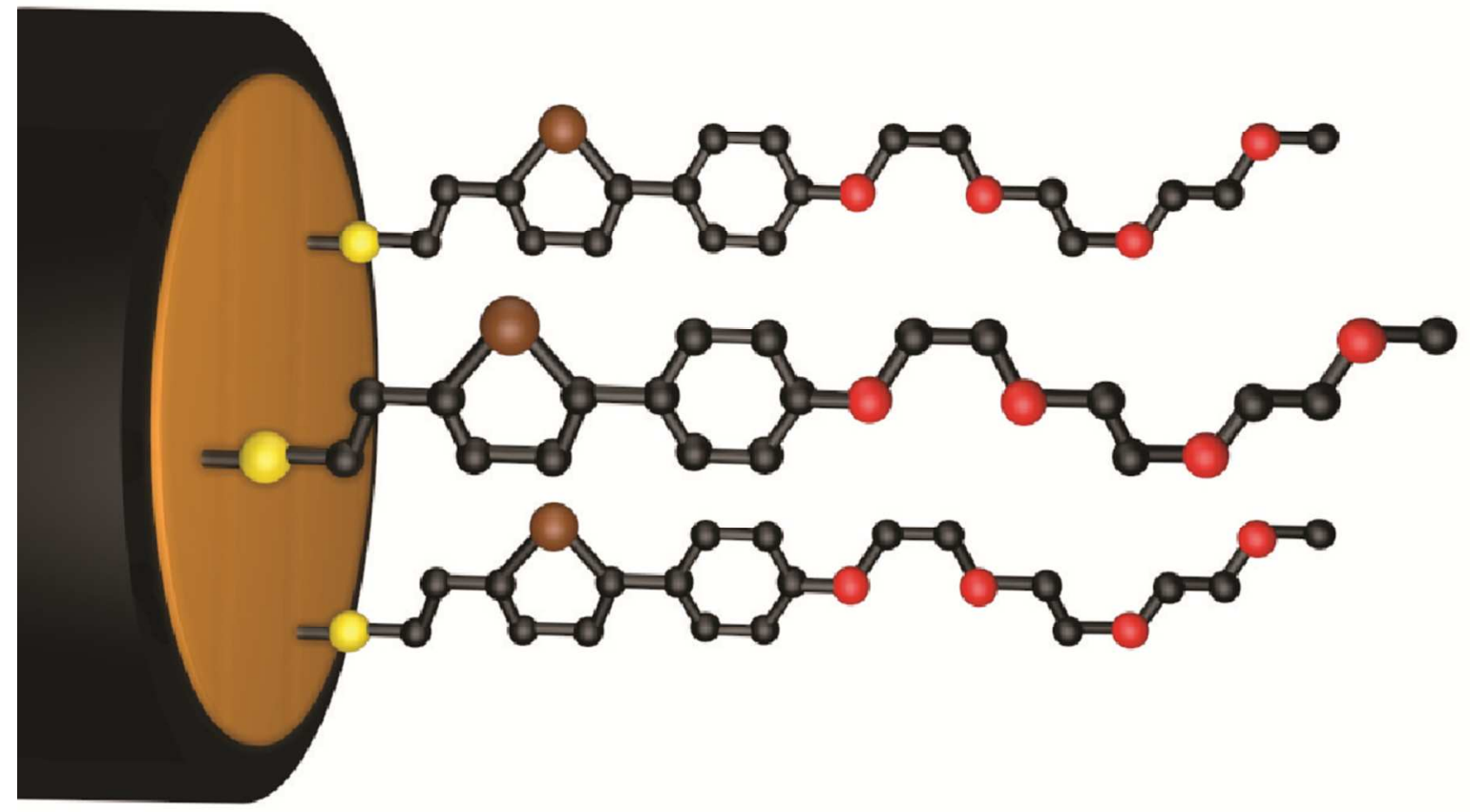

Figures and Schemes

Figure 1. (a) Structure of the thiol-modified asymmetric tellurophene used in monolayer formation studies. (b) Schematic of the tellurophene ligand covalently bonded to a gold electrode. 

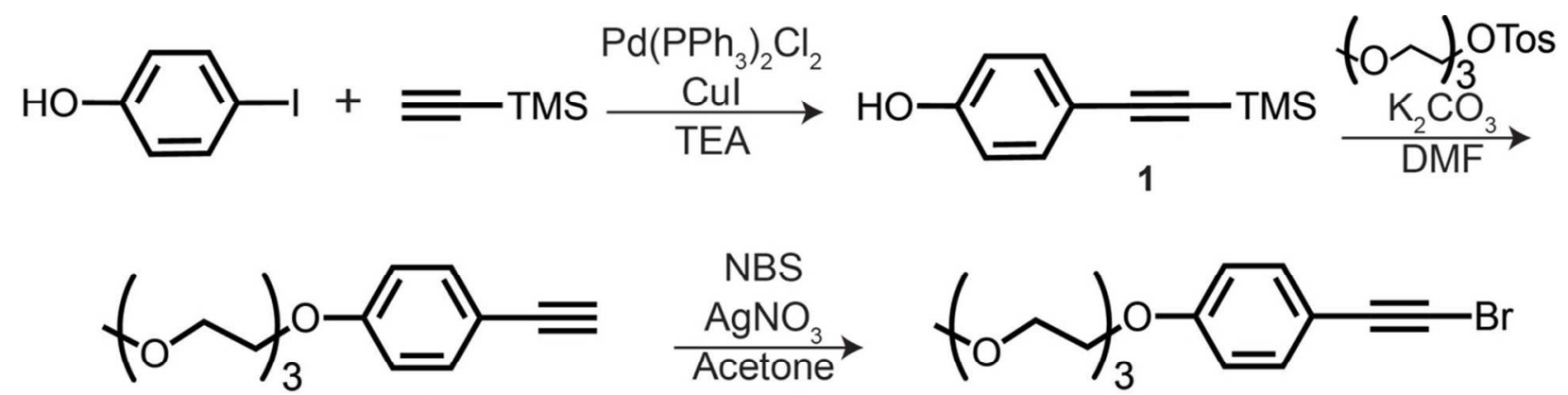

2

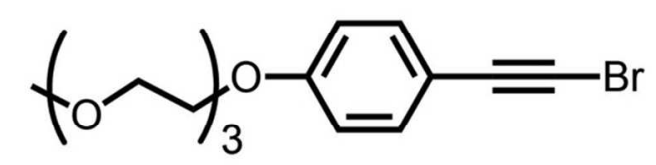

Scheme 1. Synthesis of a brominated and solubilizing alkyne coupling partner. 

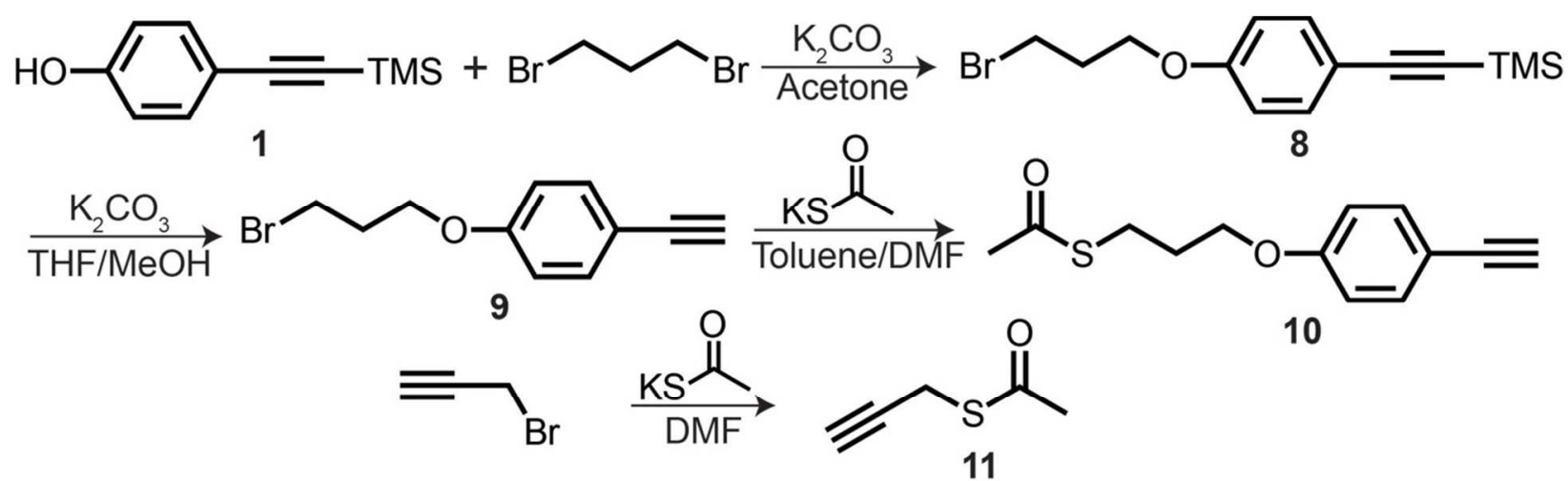

10

Scheme 2. Explored routes to thioacetate modified alkyne coupling partners, which were not accessed or not successfully coupled with $\mathbf{3}$. 

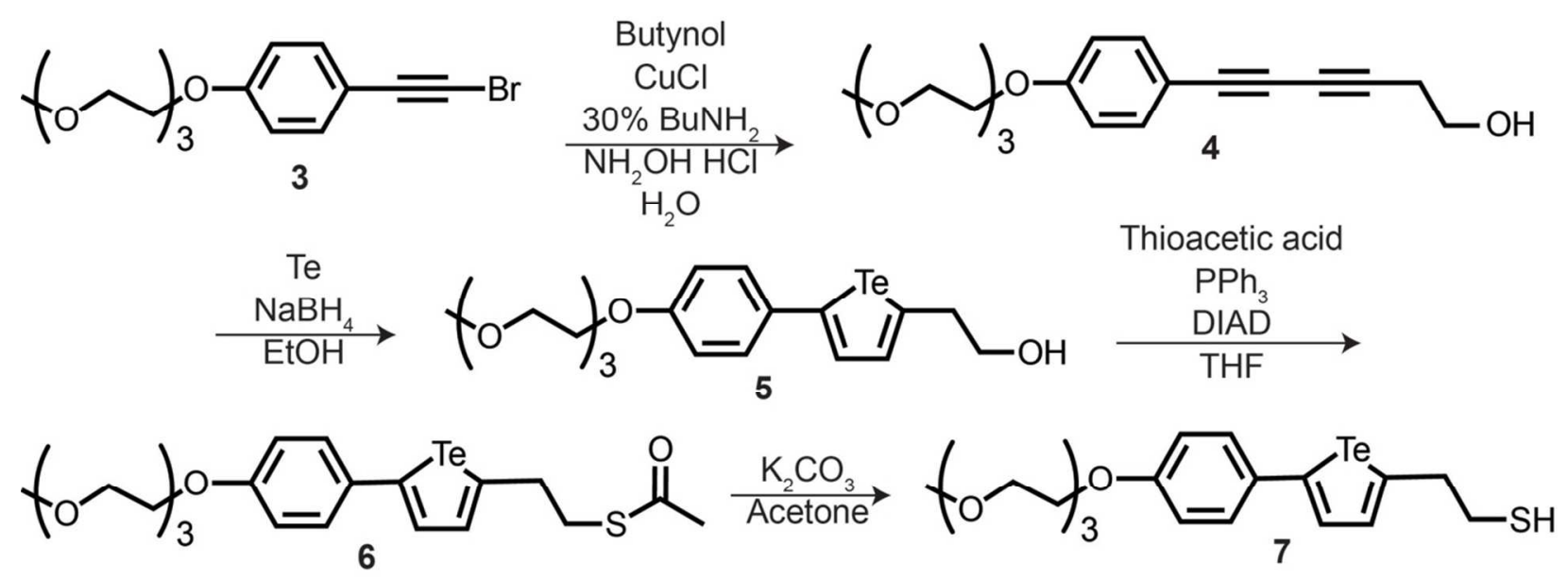

Scheme 3. Synthesis of the thiol modified tellurophene used in all electrochemical studies. 


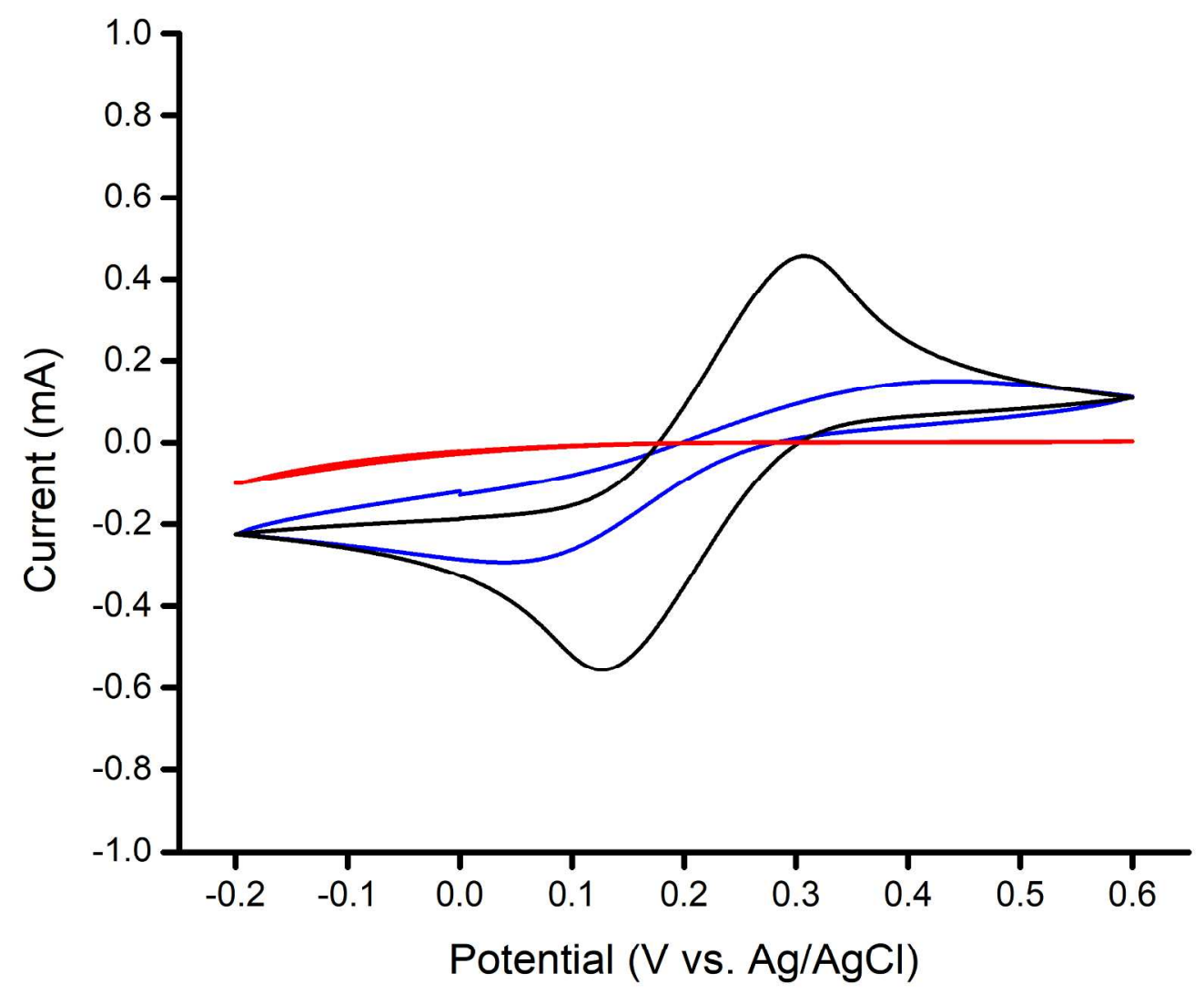

Figure 2. Cyclic voltammetry of a bare gold electrode (black) and a gold electrode with a partial (blue) and complete (red) monolayer of tellurophene ligand in $0.1 \mathrm{M} \mathrm{K}_{3} \mathrm{Fe}(\mathrm{CN})_{6}$ in water with 1 $\mathrm{M} \mathrm{KCl}$ as a supporting electrolyte. Scan rate: $100 \mathrm{mV} / \mathrm{s}$. 


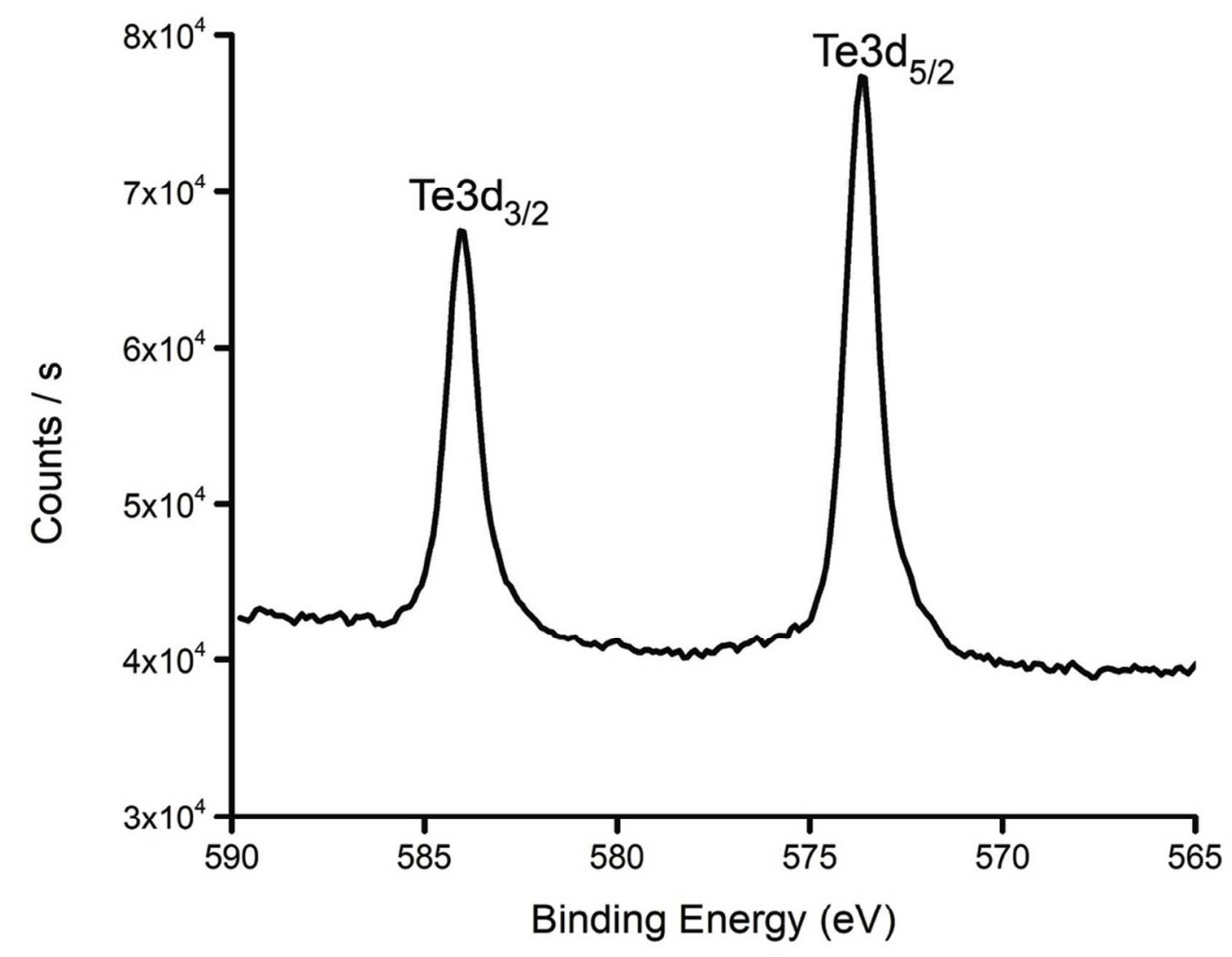

Figure 3. XPS measurement confirming the presence of tellurium on a gold-coated silicon substrate after monolayer formation. 

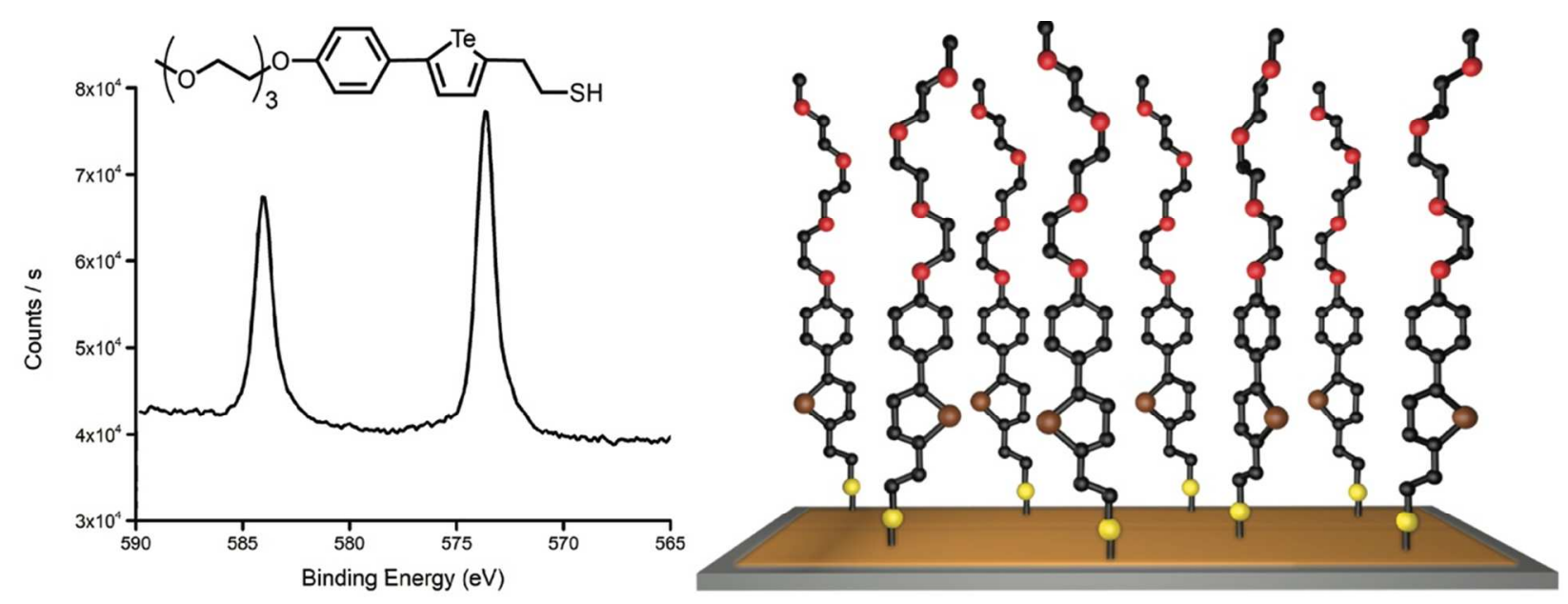

\section{Graphical Abstract}

